\title{
On the Mortality Problem: \\ From Multiplicative Matrix Equations to Linear Recurrence Sequences and Beyond
}

\author{
Paul C. Bell \\ Department of Computer Science, Byrom Street, Liverpool John Moores University, \\ Liverpool, L3-3AF, UK \\ p.c.bell@ljmu.ac.uk \\ Igor Potapov \\ Department of Computer Science, Ashton Building, Ashton Street, \\ University of Liverpool, Liverpool, L69-3BX, UK \\ potapov@liverpool.ac.uk

\section{Pavel Semukhin} \\ Department of Computer Science, University of Oxford, Wolfson Building, \\ Parks Road, Oxford, OX1 3QD, UK \\ pavel.semukhin@cs.ox.ac.uk
}

\begin{abstract}
We consider the following variant of the Mortality Problem: given $k \times k$ matrices $A_{1}, A_{2}, \ldots, A_{t}$, does there exist nonnegative integers $m_{1}, m_{2}, \ldots, m_{t}$ such that the product $A_{1}^{m_{1}} A_{2}^{m_{2}} \cdots A_{t}^{m_{t}}$ is equal to the zero matrix? It is known that this problem is decidable when $t \leq 2$ for matrices over algebraic numbers but becomes undecidable for sufficiently large $t$ and $k$ even for integral matrices.

In this paper, we prove the first decidability results for $t>2$. We show as one of our central results that for $t=3$ this problem in any dimension is Turing equivalent to the well-known Skolem problem for linear recurrence sequences. Our proof relies on the Primary Decomposition Theorem for matrices that was not used to show decidability results in matrix semigroups before. As a corollary we obtain that the above problem is decidable for $t=3$ and $k \leq 3$ for matrices over algebraic numbers and for $t=3$ and $k=4$ for matrices over real algebraic numbers. Another consequence is that the set of triples $\left(m_{1}, m_{2}, m_{3}\right)$ for which the equation $A_{1}^{m_{1}} A_{2}^{m_{2}} A_{3}^{m_{3}}$ equals the zero matrix is equal to a finite union of direct products of semilinear sets.

For $t=4$ we show that the solution set can be non-semilinear, and thus it seems unlikely that there is a direct connection to the Skolem problem. However we prove that the problem is still decidable for upper-triangular $2 \times 2$ rational matrices by employing powerful tools from transcendence theory such as Baker's theorem and S-unit equations.
\end{abstract}

2012 ACM Subject Classification Theory of computation $\rightarrow$ Computability; Mathematics of computing $\rightarrow$ Computations on matrices

Keywords and phrases Linear recurrence sequences, Skolem's problem, mortality problem, matrix equations, primary decomposition theorem, Baker's theorem

Digital Object Identifier 10.4230/LIPIcs.MFCS.2019.83

Related Version A full version of the paper is available at https://arxiv.org/abs/1902.10188.

Funding Igor Potapov: Partially supported by EPSRC grants EP/R018472/1 and EP/M00077X/1. Pavel Semukhin: Supported by ERC grant AVS-ISS (648701).

Acknowledgements We thank Prof. James Worrell for useful discussions, particularly related to S-unit equations. 


\section{Introduction}

A large number of naturally defined matrix problems are still unanswered, despite the long history of matrix theory. Some of these questions have recently drawn renewed interest in the context of the analysis of digital processes, verification problems, and links with several fundamental questions in mathematics $[11,7,37,39,38,35,17,14,15,36,5,41,27]$.

One of these challenging problems is the Mortality Problem of whether the zero matrix belongs to a finitely generated matrix semigroup. It plays a central role in many questions from control theory and software verification $[44,10,8,36,2]$. The mortality problem has been known to be undecidable for matrices in $\mathbb{Z}^{3 \times 3}$ since 1970 [40] and the current undecidability bounds for the $M(d, k \times k)$ problem (i.e. the mortality problem for semigroups generated by $d$ matrices of size $k \times k)$ are $M(6,3 \times 3), M(4,5 \times 5), M(3,9 \times 9)$ and $M(2,15 \times 15)$, see [13]. It is also known that the problem is NP-hard for $2 \times 2$ integer matrices [4] and is decidable for $2 \times 2$ integer matrices with determinant $0, \pm 1$ [34]. In the case of finite matrix semigroups of any dimension the mortality problem is known to be PSPACE-complete [26].

In this paper, we study a very natural variant of the mortality problem when matrices must appear in a fixed order (i.e. under bounded language constraint): Given $k \times k$ matrices $A_{1}, A_{2}, \ldots, A_{t}$ over a ring $\mathcal{F}$, do there exist $m_{1}, m_{2}, \ldots, m_{t} \in \mathbb{N}$ such that $A_{1}^{m_{1}} A_{2}^{m_{2}} \ldots A_{t}^{m_{t}}=$ $\mathbf{O}_{k, k}$, where $\mathbf{O}_{k, k}$ is $k \times k$ zero matrix?

In general (i.e. replacing $\mathbf{O}_{k, k}$ by other matrices) this problem is known as the solvability of multiplicative matrix equations and has been studied for many decades. In its simplest form, when $k=1$, the problem was studied by Harrison in 1969 [21] as a reformulation of the "accessibility problem" for linear sequential machines. The case $t=1$ was solved in polynomial time in a celebrated paper by Kannan and Lipton in 1980 [25]. The case $t=2$, i.e. $A^{x} B^{y}=C$ where $A, B$ and $C$ are commuting matrices was solved by Cai, Lipton and Zalcstein in 1994 [12]. Later, in 1996, the solvability of matrix equations over commuting matrices was solved in polynomial time in [1] and in 2010 it was shown in [3] that $A^{x} B^{y}=C$ is decidable for non-commuting matrices of any dimension with algebraic coefficients by a reduction to the commutative case from [1]. However, it was also shown in [3] that the solvability of multiplicative matrix equations for sufficiently large natural numbers $t$ and $k$ is in general undecidable by an encoding of Hilbert's tenth problem and in particular for the mortality problem with bounded language constraint. In 2015 it was also shown that the undecidability result holds for such equations with unitriangluar matrices [31] and also in the case of specific equations with nonnegative matrices [23].

The decidability of matrix equations for non-commuting matrices is only known as corollaries of either recent decidability results for solving membership problem in $2 \times 2$ matrix semigroups $[41,42]$ or in the case of quite restricted classes of matrices, e.g. matrices from the Heisenberg group [27, 28] or row-monomial matrices over commutative semigroups [30]. In the other direction, progress has been made for matrix-exponential equations, but again in the case of commuting matrices [36].

In this paper, we prove the first decidability results for the above problem when $t=3$ and $t=4$. We will call these problems the ABC-Z and ABCD-Z problems, respectively. More precisely, we will show that the ABC-Z problem in any dimension is Turing equivalent to the Skolem problem (also known as Skolem-Pisot problem) which asks whether a given linear recurrence sequence ever reaches zero. Our proof relies on the Primary Decomposition Theorem for matrices (Theorem 2) that was not used to show decidability results in matrix semigroups before. As a corollary, we obtain that the ABC-Z problem is decidable for $2 \times 2$ and $3 \times 3$ matrices over algebraic numbers and also for $4 \times 4$ matrices over real algebraic 
numbers. Another consequence of the above equivalence is that the set of triples $(m, n, \ell)$ that satisfy the equation $A^{m} B^{n} C^{\ell}=\mathbf{O}_{k \times k}$ can be expressed as a finite union of direct products of semilinear sets.

In contrast to the $\mathrm{ABC}-\mathrm{Z}$ problem, we show that the solution set of the ABCD-Z problem can be non-semilinear. This indicates that the ABCD-Z problem is unlikely to be related to the Skolem problem. However we will show that the ABCD-Z problem is decidable for upper-triangular $2 \times 2$ rational matrices. The proof of this result relies on powerful tools from transcendence theory such as Baker's theorem for linear forms in logarithms, S-unit equations from algebraic number theory and the Frobenius rank inequality from matrix analysis. More precisely, we will reduce the ABCD-Z equation for upper-triangular $2 \times 2$ rational matrices to an equation of the form $a x+b y+c z=0$, where $x, y, z$ are S-units, and then use an upper bound on the solutions of this equation (as in Theorem 5). On the other hand, if we try to generalize this result to arbitrary $2 \times 2$ rational matrices or to upper-triangular matrices of higher dimension, then we end up with an equation that contain a sum of four or more S-units, and for such equations no effective upper bounds on their solutions are known. So, these generalizations seems to lie beyond the reach of current mathematical knowledge.

\section{Preliminaries}

We denote by $\mathbb{N}, \mathbb{Z}, \mathbb{Q}$ and $\mathbb{C}$ the sets of natural, integer, rational and complex numbers, respectively. Further, we denote by $\mathbf{A}$ the set of algebraic numbers and by $\mathbf{A}_{\mathbb{R}}$ the set of real algebraic numbers.

For a prime number $p$ we define a valuation $v_{p}(x)$ for nonzero $x \in \mathbb{Q}$ as follows: if $x=p^{k} \frac{m}{n}$, where $m, n \in \mathbb{Z}$ and $p$ does not divide $m$ or $n$, then $v_{p}(x)=v_{p}\left(p^{k} \frac{m}{n}\right)=k$.

Throughout this paper $\mathcal{F}$ will denote either the ring of integers $\mathbb{Z}$ or one of the fields $\mathbb{Q}$, $\mathbf{A}, \mathbf{A}_{\mathbb{R}}$ or $\mathbb{C}$. We will use the notation $\mathcal{F}^{n \times m}$ for the set of $n \times m$ matrices over $\mathcal{F}$.

We denote by $\mathbf{e}_{i}$ the $i$ 'th standard basis vector of some dimension (which will be clear from the context). Let $\mathbf{O}_{n, m}$ be the zero matrix of size $n \times m, \mathbf{I}_{n}$ be the identity matrix of size $n \times n$, and $\mathbf{0}_{n}$ be the zero column vector of length $n$. Given a finite set of matrices $\mathcal{G} \subseteq \mathcal{F}^{n \times n}$, we denote by $\langle\mathcal{G}\rangle$ the multiplicative semigroup generated by $\mathcal{G}$.

If $A \in \mathcal{F}^{m \times m}$ and $B \in \mathcal{F}^{n \times n}$, then we define their direct sum as $A \oplus B=\left[\begin{array}{c|l}A & \mathbf{O}_{m, n} \\ \hline \mathbf{O}_{n, m} & B\end{array}\right]$. Let $C \in \mathcal{F}^{k \times k}$ be a square matrix. We write $\operatorname{det}(C)$ for the determinant of $C$. We call $C$ singular if $\operatorname{det}(C)=0$, otherwise it is said to be invertible (or non-singular). Matrices $A$ and $B$ from $\mathcal{F}^{k \times k}$ are called similar if there exists an invertible $k \times k$ matrix $S$ (perhaps over a larger field containing $\mathcal{F}$ ) such that $A=S B S^{-1}$. In this case, $S$ is said to be a similarity matrix transforming $A$ to $B$.

We will also require the following inequality regarding ranks of matrices, known as the Frobenius rank inequality, see [24] for further details.

- Theorem 1 (Frobenius Rank Inequality). Let $A, B, C \in \mathcal{F}^{k \times k}$. Then

$$
R k(A B)+R k(B C) \leq R k(A B C)+R k(B)
$$

In the proof of our first main result about the ABC-Z problem we will make use of the primary decomposition theorem for matrices.

- Theorem 2 (Primary Decomposition Theorem [22]). Let A be a matrix from $\mathcal{F}^{n \times n}$, where $\mathcal{F}$ is a field. Let $m_{A}(x)$ be the minimal polynomial for A such that

$$
m_{A}(x)=p_{1}(x)^{r_{1}} \cdots p_{k}(x)^{r_{k}}
$$


where the $p_{i}(x)$ are distinct irreducible monic polynomials over $\mathcal{F}$ and the $r_{i}$ are positive integers. Let $W_{i}$ be the null space of $p_{i}(A)^{r_{i}}$ and let $S_{i}$ be a basis for $W_{i}$. Then

(1) $S_{1} \cup \cdots \cup S_{k}$ is a basis for $\mathcal{F}^{n}$ and $\mathcal{F}^{n}=W_{1} \oplus \cdots \oplus W_{k}$,

(2) each $W_{i}$ is invariant under $A$, that is, $A \mathbf{x} \in W_{i}$ for any $\mathbf{x} \in W_{i}$,

(3) let $S$ be a matrix whose columns are equal to the basis vectors from $S_{1} \cup \cdots \cup S_{k}$; then

$$
S^{-1} A S=A_{1} \oplus \cdots \oplus A_{k},
$$

where each $A_{i}$ is a matrix over $\mathcal{F}$ of the size $\left|S_{i}\right| \times\left|S_{i}\right|$, and the minimal polynomial of $A_{i}$ is equal to $p_{i}(x)^{r_{i}}$.

The next two propositions are well-known facts.

- Proposition 3. If $p(x)$ is a polynomial over a field $\mathcal{F}$, where $\mathcal{F}$ is either $\mathbb{Q}, \mathbf{A}$ or $\mathbf{A}_{\mathbb{R}}$, then the primary decomposition of $p(x)$ can be algorithmically computed.

- Proposition 4. Let $A \in \mathcal{F}^{n \times n}$ and $m_{A}(x)$ be the minimal polynomial of $A$. Then $A$ is invertible if and only if $m_{A}(x)$ has nonzero free coefficient, i.e., $m_{A}(x)$ is not divisible by $x$.

Our proof of the decidability of ABCD-Z problem for $2 \times 2$ upper-triangular rational matrices relies on the following result which is proved using Baker's theorem on linear forms in logarithms (see Corollary 4 in [16] and also [18]).

- Theorem 5. Let $S=\left\{p_{1}, \ldots, p_{s}\right\}$ be a finite collection of prime numbers and let $a, b, c$ be relatively prime nonzero integers, that is, $\operatorname{gcd}(a, b, c)=1$.

If $x, y, z$ are relatively prime nonzero integers composed of primes from $S$ that satisfy the equation $a x+b y+c z=0$, then

$$
\max \{|x|,|y|,|z|\}<\exp \left(s^{C s} P^{4 / 3} \log A\right)
$$

for some constant $C$, where $P=\max \left\{p_{1}, \ldots, p_{s}\right\}$ and $A=\max \{|a|,|b|,|c|, 3\}$.

- Remark. Rational numbers whose numerator and denominator are divisible only by the primes from $S$ are called $S$-units.

\section{Linear recurrence sequences and semilinear sets}

There is a long history in computer science and mathematics of studying sequences of numbers defined by some recurrence relation, where the next value in the sequence depends upon some "finite memory" of previous values in the sequence. Possibly the simplest, and certainly the most well known of these, is the Fibonacci sequence, which may be defined by the recurrence $F(n)=F(n-1)+F(n-2)$ with $F(0)=F(1)=1$ being given as the initial conditions of the sequence. We may generalise the Fibonacci sequence to define a linear recurrence sequence, which find application in many areas of mathematics and other sciences and for which many questions remain open. Let $\mathcal{F}$ be a ring; a sequence $\left(u_{n}\right)_{n=0}^{\infty}$ is called a linear recurrence sequence (1-LRS) if it satisfies a relation of the form:

$$
u_{n}=a_{k-1} u_{n-1}+\cdots+a_{1} u_{n-k+1}+a_{0} u_{n-k},
$$

for any $n \geq k$, where each $a_{0}, a_{1}, \ldots, a_{k-1} \in \mathcal{F}$ are fixed coefficients ${ }^{1}$. Such a sequence $\left(u_{n}\right)_{n=0}^{\infty}$ is said to be of depth $k$ if it satisfies no shorter linear recurrence relation (for any

1 In the literature, such a sequence is ordinarily called an LRS; we use the nomenclature 1-LRS since we will study a multidimensional variant of this concept. Also, 1-LRS are usually considered over integers, but in the present paper we will consider such sequences over algebraic numbers. 
$k^{\prime}<k$ ). We call the initial $k$ values of the sequence $u_{0}, u_{1}, \ldots, u_{k-1}$ the initial conditions of the 1-LRS. Given the initial conditions and coefficients of a 1-LRS, every element is uniquely determined.

The zero set of a 1-LRS is defined as follows: $\mathcal{Z}\left(u_{n}\right)=\left\{j \in \mathbb{N} \mid u_{j}=0\right\}$.

There are various questions that one may ask regarding $\mathcal{Z}\left(u_{n}\right)$. One notable example relates to the famous "Skolem's problem" which is stated in the following way:

- Problem 6 (Skolem's Problem). Given the coefficients and initial conditions of a depth $k$ $1-L R S\left(u_{n}\right)_{n=0}^{\infty}$, determine if $\mathcal{Z}\left(u_{n}\right)$ is the empty set.

Skolem's problem has a long and rich history, see [19] for a good survey. We note here that the problem remains open despite properties of zero sets having been studied even since 1934 [43]. It is known that the Skolem problem is at least NP-hard [9] and that it is decidable for depth 3 over $\mathbf{A}$ and for depth 4 over $\mathbf{A}_{\mathbb{R}}$, see [44] and [33] . Other interesting questions are related to the structure of $\mathcal{Z}\left(u_{n}\right)$. We remind the reader the definition of semilinear sets.

- Definition 7 (Semilinear set). A set $S \subseteq \mathbb{N}$ is called semilinear if it is the union of a finite set and finitely many arithmetic progressions.

A seminal result regarding 1-LRSs is that there zero sets are semilinear.

- Theorem 8 (Skolem, Mahler, Lech [32, 43, 29] and [19, 20]). The zero set of a 1-LRS over $\mathbb{C}$ (or more generally over any field of characteristic 0) is semilinear.

In particular, if $\left(u_{n}\right)_{n=0}^{\infty}$ is a 1-LRS whose coefficients and initial conditions are algebraic numbers, then one can algorithmically find a number $L \in \mathbb{N}$ such that for every $i=0, \ldots, L-1$, if we let $u_{m}^{i}=u_{i+m L}$, then

(1) the sequence $\left(u_{m}^{i}\right)_{m=0}^{\infty}$ is a 1-LRS of the same depth as $\left(u_{n}\right)_{n=0}^{\infty}$, and

(2) either $\mathcal{Z}\left(u_{m}^{i}\right)=\mathbb{N}$ or $\mathcal{Z}\left(u_{m}^{i}\right)$ is finite.

Note that in the above theorem we can decide whether $\mathcal{Z}\left(u_{m}^{i}\right)$ is finite or $\mathcal{Z}\left(u_{m}^{i}\right)=\mathbb{N}$ because $\mathcal{Z}\left(u_{m}^{i}\right)=\mathbb{N}$ if and only if $u_{0}^{i}=\cdots=u_{k-1}^{i}=0$, where $k$ is the depth of $\left(u_{m}^{i}\right)_{m=0}^{\infty}$.

We will also consider a stronger version of the Skolem problem.

- Problem 9 (Strong Skolem's Problem). Given the coefficients and initial conditions of a 1-LRS $\left(u_{n}\right)_{n=0}^{\infty}$ over $\mathbf{A}$, find a description of the set $\mathcal{Z}\left(u_{n}\right)$. That is, find a finite set $F$ such that $\mathcal{Z}\left(u_{n}\right)=F$ if $\mathcal{Z}\left(u_{n}\right)$ is finite or, if $\mathcal{Z}\left(u_{n}\right)$ is infinite, find a finite set $F$, a constant $L \in \mathbb{N}$ and numbers $i_{1}, \ldots, i_{t} \in\{0, \ldots, L-1\}$ such that

$$
\mathcal{Z}\left(u_{n}\right)=F \cup\left\{i_{1}+m L: m \in \mathbb{N}\right\} \cup \cdots \cup\left\{i_{t}+m L: m \in \mathbb{N}\right\} .
$$

Using the Skolem-Mahler-Lech theorem we can prove an equivalence between the strong version of the Skolem problem and the standard version ${ }^{3}$.

Theorem 10. Skolem's problem of depth $k$ over $\mathbf{A}$ is Turing equivalent to the strong Skolem's problem of the same depth.

Proof. Obviously, Skolem's problem is reducible to the strong Skolem's problem. We now show a reduction in the other direction.

Let $\left(u_{n}\right)_{n=0}^{\infty}$ be a depth- $k$ 1-LRS over A. By Theorem 8 , we can algorithmically find a number $L$ such that, for every $i=0, \ldots, L-1$, the sequence $u_{m}^{i}=u_{i+m L}$ is a 1-LRS of

\footnotetext{
2 A proof of decidability for depth 5 was claimed in [19], although there is possibly a gap in the proof [37].

3 This result was announced in [44] without a proof, probably with a similar construction in mind.
} 
depth $k$ which is either everywhere zero, that is, $\mathcal{Z}\left(u_{m}^{i}\right)=\mathbb{N}$ or $\mathcal{Z}\left(u_{m}^{i}\right)$ is finite. Recall that we can decide whether $\mathcal{Z}\left(u_{m}^{i}\right)$ is equal to $\mathbb{N}$ by considering the first $k$ terms of $\left(u_{m}^{i}\right)_{m=0}^{\infty}$.

By definition, we have $\mathcal{Z}\left(u_{n}\right)=\bigcup_{i=0}^{L-1}\left\{i+L \mathcal{Z}\left(u_{m}^{i}\right)\right\}$. So, if $\mathcal{Z}\left(u_{m}^{i}\right)=\mathbb{N}$, then $\left\{i+L \mathcal{Z}\left(u_{m}^{i}\right)\right\}=$ $\{i+m L: m \in \mathbb{N}\}$, and if $\mathcal{Z}\left(u_{m}^{i}\right)$ is finite, then so is $\left\{i+L \cdot \mathcal{Z}\left(u_{m}^{i}\right)\right\}$.

To finish the proof we need to show how to compute $\mathcal{Z}\left(u_{m}^{i}\right)$, and hence $\left\{i+L \cdot \mathcal{Z}\left(u_{m}^{i}\right)\right\}$, when it is finite. For this we will use an oracle for the Skolem problem. Let $m^{\prime}$ be the smallest number such that $\mathcal{Z}\left(u_{m+m^{\prime}}^{i}\right)$ is empty. Such $m^{\prime}$ exists because $\mathcal{Z}\left(u_{m}^{i}\right)$ is finite. Furthermore, $\left(u_{m+m^{\prime}}\right)_{m=0}^{\infty}$ is a 1-LRS of depth $k$ for any $m^{\prime}$. So, we ask the oracle for the Skolem problem to decide whether $\mathcal{Z}\left(u_{m+m^{\prime}}^{i}\right)=\emptyset$ for each $m^{\prime} \in \mathbb{N}$ starting from 0 until we find one for which $\mathcal{Z}\left(u_{m+m^{\prime}}^{i}\right)$ is empty. Note that we do not have any bound on $m^{\prime}$ because we do not even know the size of $\mathcal{Z}\left(u_{m}^{i}\right)$. All we know is that $\mathcal{Z}\left(u_{m}^{i}\right)$ is finite, and hence the above algorithm will eventually terminate. Since $\mathcal{Z}\left(u_{m}^{i}\right)$ is a subset of $\left\{0, \ldots, m^{\prime}\right\}$, then we can compute it by checking whether $u_{m}^{i}=0$ for $m=0, \ldots, m^{\prime}$.

Linear recurrence sequences can also be represented using matrices [19]:

- Lemma 11. Let $\mathcal{F}$ be a ring; for a sequence $\left(u_{n}\right)_{n=0}^{\infty}$ over $\mathcal{F}$ the following are equivalent: (1) $\left(u_{n}\right)_{n=0}^{\infty}$ is a 1-LRS of depth $k$.

(2) There are vectors $\mathbf{u}, \mathbf{v} \in \mathcal{F}^{k}$ and a matrix $M \in \mathcal{F}^{k \times k}$ such that $u_{n}=\mathbf{u}^{T} M^{n} \mathbf{v}$ for $n \in \mathbb{N}$.

Moreover, for any matrix $M \in \mathcal{F}^{k \times k}$, the sequence $u_{n}=\left(M^{n}\right)_{[1, k]}$ is a 1-LRS of depth at most $k$. On the other hand, if $\left(u_{n}\right)_{n=0}^{\infty}$ is a 1-LRS of depth $k$, then there is a matrix $M \in \mathcal{F}^{(k+1) \times(k+1)}$ such that $u_{n}=\left(M^{n}\right)_{[1, k+1]}$ for all $n \in \mathbb{N}$.

Lemma 11 motivates the following definition of $n$-dimensional Linear Recurrence Sequences ( $n$-LRSs) which as we show later are related to the mortality problem for bounded languages.

- Definition 12 (n-LRS). A multidimensional sequence $u_{m_{1}, m_{2}, \ldots, m_{n}}$ is called an $n$-LRS of depth $k$ over $\mathcal{F}$ if there exist two vectors $\mathbf{u}, \mathbf{v} \in \mathcal{F}^{k}$ and matrices $M_{1}, M_{2}, \ldots, M_{n} \in \mathcal{F}^{k \times k}$ such that

$$
u_{m_{1}, m_{2}, \ldots, m_{n}}=\mathbf{u}^{T} M_{1}^{m_{1}} M_{2}^{m_{2}} \cdots M_{n}^{m_{n}} \mathbf{v} .
$$

\section{$4 \quad$ The mortality problem for bounded languages}

We remind the reader the definition of the mortality problem for bounded languages.

- Problem 13 (Mortality for bounded languages). Given $k \times k$ matrices $A_{1}, \ldots, A_{t}$ over a ring $\mathcal{F}$, do there exist $m_{1}, m_{2}, \ldots, m_{t} \in \mathbb{N}$ such that

$$
A_{1}^{m_{1}} A_{2}^{m_{2}} \ldots A_{t}^{m_{t}}=\mathbf{O}_{k, k} .
$$

Recall that for $t=3$ and $t=4$ this problem is called the ABC-Z and ABCD-Z problem, respectively. Our first main result is that the ABC-Z problem is computationally equivalent to the Skolem problem for 1-LRS. Our reduction holds in any dimension and over the same number field which means that any new decidability results for the Skolem problem will automatically extend the decidability of ABC-Z equations and can immediately lead to new decidability results for equations in dimensions 2, 3 and 4 . For the proof we will need the following technical lemma. 
- Lemma 14. Let $\mathcal{F}$ be a field, and suppose $A, B, C \in \mathcal{F}^{k \times k}$ are matrices of the form

$$
A=\left[\begin{array}{c|c}
A_{s, s} & \mathbf{O}_{s, k-s} \\
\hline \mathbf{O}_{k-s, s} & \mathbf{O}_{k-s, k-s}
\end{array}\right], B=\left[\begin{array}{c|c}
B_{s, t} & X_{s, k-t} \\
\hline Y_{k-s, t} & Z_{k-s, k-t}
\end{array}\right], C=\left[\begin{array}{c|c}
C_{t, t} & \mathbf{O}_{t, k-t} \\
\hline \mathbf{O}_{k-t, t} & \mathbf{O}_{k-t, k-t}
\end{array}\right]
$$

for some $s, t \leq k$, where $A_{s, s}, B_{s, t}, X_{s, k-t}, Y_{k-s, t}, Z_{k-s, k-t}$ and $C_{t, t}$ are matrices over $\mathcal{F}$ whose dimensions are indicated by their subscripts (in particular, $A=A_{s, s} \oplus \mathbf{O}_{k-s, k-s}$ and $\left.C=C_{t, t} \oplus \mathbf{O}_{k-t, k-t}\right)$. If $A_{s, s}$ and $C_{t, t}$ are invertible matrices, then the equation $A B C=\mathbf{O}_{k, k}$ is equivalent to $B_{s, t}=\mathbf{O}_{s, t}$.

Proof. It is not hard to check that

$$
A B=\left[\begin{array}{c|c}
A_{s, s} & \mathbf{O}_{s, k-s} \\
\hline \mathbf{O}_{k-s, s} & \mathbf{O}_{k-s, k-s}
\end{array}\right] \cdot\left[\begin{array}{c|c}
B_{s, t} & X_{s, k-t} \\
\hline Y_{k-s, t} & Z_{k-s, k-t}
\end{array}\right]=\left[\begin{array}{c|c}
A_{s, s} B_{s, t} & A_{s, s} X_{s, k-t} \\
\hline \mathbf{O}_{k-s, t} & \mathbf{O}_{k-s, k-t}
\end{array}\right],
$$

and hence

$$
(A B) C=\left[\begin{array}{c|c}
A_{s, s} B_{s, t} & A_{s, s} X_{s, k-t} \\
\hline \mathbf{O}_{k-s, t} & \mathbf{O}_{k-s, k-t}
\end{array}\right] \cdot\left[\begin{array}{c|c}
C_{t, t} & \mathbf{O}_{t, k-t} \\
\hline \mathbf{O}_{k-t, t} & \mathbf{O}_{k-t, k-t}
\end{array}\right]=\left[\begin{array}{c|c}
A_{s, s} B_{s, t} C_{t, t} & \mathbf{O}_{s, k-t} \\
\hline \mathbf{O}_{k-s, t} & \mathbf{O}_{k-s, k-t}
\end{array}\right] .
$$

So, if $B_{s, t}=\mathbf{O}_{s, t}$, then $A B C=\mathbf{O}_{k, k}$. Conversely, if $A B C=\mathbf{O}_{k, k}$, then $A_{s, s} B_{s, t} C_{t, t}=\mathbf{O}_{s, t}$. Using the fact that $A_{s, s}$ and $C_{t, t}$ are invertible matrices, we can multiply the equation $A_{s, s} B_{s, t} C_{t, t}=\mathbf{O}_{s, t}$ by $A_{s, s}^{-1}$ on the left and by $C_{t, t}^{-1}$ on the right to obtain that $B_{s, t}=\mathbf{O}_{s, t}$.

Theorem 15. Let $\mathcal{F}$ be the ring of integers $\mathbb{Z}$ or one of the fields $\mathbb{Q}$, $\mathbf{A}$ or $\mathbf{A}_{\mathbb{R}}$. Then the $A B C-Z$ problem for matrices from $\mathcal{F}^{k \times k}$ is Turing equivalent to the Skolem problem of depth $k$ over $\mathcal{F}$.

Proof. First, we show reduction from the ABC-Z problem to the Skolem problem.

Clearly, the ABC-Z problem over $\mathbb{Z}$ is equivalent to the $\mathrm{ABC}-\mathrm{Z}$ problem over $\mathbb{Q}$ (by multiplying the matrices $A, B, C$ by a suitable integer number). It is also not hard to see that the Skolem problem for 1-LRS over $\mathbb{Q}$ is equivalent to the Skolem problem over $\mathbb{Z}$ for 1 -LRS of the same depth. Indeed, by Lemma 11 we can express any 1-LRS $\left(u_{n}\right)_{n=0}^{\infty}$ over $\mathbb{Q}$ as $u_{n}=\mathbf{u}^{T} M^{n} \mathbf{v}$ for some rational vectors $\mathbf{u}$ and $\mathbf{v}$ and a rational matrix $M$. If we multiply $\mathbf{u}, \mathbf{v}$ and $M$ by a suitable natural number $t$, then $\left(t^{n+2} u_{n}\right)_{n=0}^{\infty}$ will be an integer 1-LRS, which has the same zero set as $\left(u_{n}\right)_{n=0}^{\infty}$. Hence, without loss of generality, we will assume that $\mathcal{F}$ is one of the fields $\mathbb{Q}, \mathbf{A}$ or $\mathbf{A}_{\mathbb{R}}$.

Consider an instance of the ABC-Z problem: $A^{m} B^{n} C^{\ell}=\mathbf{O}_{k, k}$, where $A, B, C \in \mathcal{F}^{k, k}$. Let $\chi_{A}(x)$ be the characteristic polynomial of $A$. By Proposition 3, we can find a primary decomposition $\chi_{A}(x)=p_{1}(x)^{m_{1}} \cdots p_{t}(x)^{m_{t}}$, where $p_{1}(x), \ldots, p_{t}(x)$ are distinct irreducible monic polynomials. From this decomposition we can find the minimal polynomial $m_{A}(x)$ of $A$ because $m_{A}(x)$ is a factor of $\chi_{A}(x)$, and we can check all divisors of $\chi_{A}(x)$ to find $m_{A}(x)$.

Let $m_{A}(x)=p_{1}(x)^{r_{1}} \cdots p_{u}(x)^{r_{u}}$, where $p_{1}(x), \ldots, p_{u}(x)$ are distinct irreducible monic polynomials. Now we apply the Primary Decomposition Theorem (Theorem 2) to $A$. Let $S_{i}$ be a basis for the null space of $p_{i}(A)^{r_{i}}$, which can be found, e.g., using Gaussian elimination. Let $S$ be a matrix whose columns are the vectors of the basis $S_{1} \cup \cdots \cup S_{u}$. Then

$$
S^{-1} A S=A_{1} \oplus \cdots \oplus A_{u}
$$

where the minimal polynomial of $A_{i}$ is $p_{i}(A)^{r_{i}}$ for $i=1, \ldots, u$. Similarly, we can compute a primary decomposition $m_{C}(x)=q_{1}(x)^{s_{1}} \cdots q_{v}(x)^{s_{v}}$ of the minimal polynomial for $C$, where $q_{1}(x), \ldots, q_{v}(x)$ are distinct irreducible monic polynomials, and a matrix $T$ such that

$$
T^{-1} C T=C_{1} \oplus \cdots \oplus C_{v}
$$

where the minimal polynomial of $C_{i}$ is $q_{i}(C)^{s_{i}}$ for $i=1, \ldots, v$. 
Note that if $p(x)$ is an irreducible monic polynomial, then either $p(x)=x$ or $x$ does not divide $p(x)$. So, among the polynomials $p_{1}(x), \ldots, p_{u}(x)$ in the primary decomposition of $m_{A}(x)$ at most one is equal to $x$, and the same holds for the polynomials $q_{1}(x), \ldots, q_{v}(x)$ in the primary decomposition of $m_{C}(x)$.

Suppose, for example, that $p_{u}(x)=x$. In this case $m_{A}(x)=p_{1}(x)^{r_{1}} \cdots p_{u-1}(x)^{r_{u-1}} x^{r_{u}}$, and $S^{-1} A S=A_{1} \oplus \cdots \oplus A_{u-1} \oplus A_{u}$, where the minimal polynomial of $A_{u}$ is $x^{r_{u}}$, and hence $A_{u}$ is a nilpotent matrix of index $r_{u}$. Recall that, for $i=1, \ldots, u-1$, the polynomial $p_{i}(x)$ is not divisible by $x$, and so is $p_{i}(x)^{r_{i}}$, which is the minimal polynomial for $A_{i}$. Hence, by Proposition $4, A_{i}$ is invertible. Let $A_{\text {inv }}=A_{1} \oplus \cdots \oplus A_{u-1}$ and $A_{\text {nil }}=A_{u}$. Then we obtain

$$
S^{-1} A S=A_{\text {inv }} \oplus A_{\text {nil }}
$$

where $A_{\mathrm{inv}}$ is invertible, and $A_{\text {nil }}$ is nilpotent. If $p_{i}(x)=x$ for some $i<u$, then we need in addition to permute some rows and columns of matrix $S$ to obtain one that gives us Equation (1) above. If none of the $p_{i}(x)$ is equal to $x$, then we assume that $A_{\text {nil }}$ is the empty matrix of size $0 \times 0$.

The same reasoning can be applied to matrix $C$, that is, we can compute an invertible matrix $C_{\text {inv }}$, a nilpotent (or empty) matrix $C_{\text {nil }}$, and an invertible matrix $T$ such that

$$
T^{-1} C T=C_{\mathrm{inv}} \oplus C_{\mathrm{nil}} \text {. }
$$

Note that the indices of the nilpotent matrices $A_{\text {nil }}$ and $C_{\text {nil }}$ are at most $k$, and hence $A_{\text {nil }}^{k}$ and $C_{\text {nil }}^{k}$ are zero (or empty) matrices.

Our goal is to find all triples $(m, n, \ell) \in \mathbb{N}^{3}$ for which $A^{m} B^{n} C^{\ell}=\mathbf{O}_{k, k}$. In order to do this we will consider four cases: (1) $m \geq k$ and $\ell \geq k$, (2) $m<k$ and $\ell<k$, (3) $m \geq k$ and $\ell<k$, and (4) $m<k$ and $\ell \geq k$.

Before dealing with each of these cases, we note that the equation $A^{m} B^{n} C^{\ell}=\mathbf{O}_{k, k}$ is equivalent to

$$
\begin{aligned}
& S\left(A_{\mathrm{inv}}^{m} \oplus A_{\mathrm{nil}}^{m}\right) S^{-1} B^{n} T\left(C_{\mathrm{inv}}^{\ell} \oplus C_{\mathrm{nil}}^{\ell}\right) T^{-1}=\mathbf{O}_{k, k} \quad \text { or to } \\
& \left(A_{\mathrm{inv}}^{m} \oplus A_{\mathrm{nil}}^{m}\right) S^{-1} B^{n} T\left(C_{\mathrm{inv}}^{\ell} \oplus C_{\mathrm{nil}}^{\ell}\right)=\mathbf{O}_{k, k}
\end{aligned}
$$

because $S$ and $T$ are invertible matrices.

Now suppose $A_{\text {inv }}$ has size $s \times s$, and $C_{\text {inv }}$ has size $t \times t$ for some $s, t \leq k$.

Case $1: \boldsymbol{m} \geq \boldsymbol{k}$ and $\ell \geq \boldsymbol{k}$. Since $m, \ell \geq k$, we have $A_{\text {nil }}^{m}=\mathbf{O}_{k-s, k-s}$ and $C_{\text {nil }}^{\ell}=$ $\mathbf{O}_{k-t, k-t}$, and hence the equation $A^{m} B^{n} C^{\ell}=\mathbf{O}_{k, k}$ is equivalent to

$$
\left(A_{\mathrm{inv}}^{m} \oplus \mathbf{O}_{k-s, k-s}\right) S^{-1} B^{n} T\left(C_{\mathrm{inv}}^{\ell} \oplus \mathbf{O}_{k-t, k-t}\right)=\mathbf{O}_{k, k} .
$$

Suppose the matrix $S^{-1} B^{n} T$ has a form $S^{-1} B^{n} T=\left[\begin{array}{c|c}B_{s, t} & X_{s, k-t} \\ \hline Y_{k-s, t} & Z_{k-s, k-t}\end{array}\right]$. Since $A_{\mathrm{inv}}^{m}$ and $C_{\mathrm{inv}}^{\ell}$ are invertible matrices, Lemma 14 implies that Equation (2) is equivalent to $B_{s, t}=\mathbf{O}_{s, t}$. Therefore, we obtain the following equivalence: $A^{m} B^{n} C^{\ell}=\mathbf{O}_{k, k}$ if and only if

$$
s_{n}^{i, j}=\left(\mathbf{e}_{i}^{\top} S^{-1}\right) B^{n}\left(T \mathbf{e}_{j}\right)=0 \text { for all } i=1, \ldots, s \text { and } j=1, \ldots, t .
$$

By Lemma 11, the sequence $\left(s_{n}^{i, j}\right)_{n=0}^{\infty}$ is a 1-LRS of order $k$ over $\mathcal{F}$. As in the proof of Theorem 10, we can use an oracle for the Skolem problem for 1-LRS of depth $k$ over $\mathcal{F}$ to compute the descriptions of the semilinear sets $\mathcal{Z}\left(s_{n}^{i, j}\right)$. Hence we can compute a description of the intersection $Z_{1}=\bigcap_{\substack{i=1, \ldots, s \\ j=1, \ldots, t}} \mathcal{Z}\left(s_{n}^{i, j}\right)$, which is also a semilinear set. An important observation is that the set $Z_{1}$ does not depend on $m$ and $\ell$. 
Below is a brief description of the remaining cases. The detailed proof of Cases 2,3 and 4 can be found in the full version [6].

Case 2: $\boldsymbol{m}<\boldsymbol{k}$ and $\ell<\boldsymbol{k}$. Fix some $m<k$ and $\ell<k$. For this particular choice of $m$ and $\ell$, we can compute a description of the semilinear set $Z_{2}(m, \ell)$ which is equal to all values of $n$ for which $A^{m} B^{n} C^{\ell}=\mathbf{O}_{k, k}$ holds for fixed $m, \ell<k$.

Case 3: $\boldsymbol{m} \geq \boldsymbol{k}$ and $\ell<\boldsymbol{k}$ and Case 4: $\boldsymbol{m}<\boldsymbol{k}$ and $\ell \geq \boldsymbol{k}$. To solve these cases we will combine ideas from Cases 1 and 2. Namely, we can compute the descriptions of the semilinear sets $Z_{3}(\ell)$ and $Z_{4}(m)$ such that

- $Z_{3}(\ell)=\left\{n: A^{m} B^{n} C^{\ell}=\mathbf{O}_{k, k}\right.$ for all $\left.m \geq k\right\}$.

- $Z_{4}(m)=\left\{n: A^{m} B^{n} C^{\ell}=\mathbf{O}_{k, k}\right.$ for all $\left.\ell \geq k\right\}$.

Combining all the above cases together, we conclude that the set of all triples $(m, n, \ell) \in \mathbb{N}^{3}$ that satisfy the equation $A^{m} B^{n} C^{\ell}=\mathbf{O}_{k, k}$ is equal to the following union

$$
\begin{gathered}
\left\{(m, n, \ell): n \in Z_{1} \text { and } m, \ell \geq k\right\} \bigcup \bigcup_{m, \ell<k}\left\{(m, n, \ell): n \in Z_{2}(m, \ell)\right\} \bigcup \\
\bigcup_{\ell<k}\left\{(m, n, \ell): n \in Z_{3}(\ell) \text { and } m \geq k\right\} \bigcup \bigcup_{m<k}\left\{(m, n, \ell): n \in Z_{4}(m) \text { and } \ell \geq k\right\} .
\end{gathered}
$$

Having a description for the above set, we can decide whether is it empty or not, that is, whether there exist $m, n, \ell \in \mathbb{N}$ such that $A^{m} B^{n} C^{\ell}=\mathbf{O}_{k, k}$.

We now show the reduction in the other direction. Let $\left(u_{n}\right)_{n=0}^{\infty}$ be a 1-LRS that satisfies a relation

$$
u_{n}=a_{k-1} u_{n-1}+\cdots+a_{1} u_{n-k+1}+a_{0} u_{n-k},
$$

where $a_{0} \neq 0$. Let $A, B$ and $C$ be the following matrices of size $k \times k$ :

$$
A=\left(\begin{array}{cccc}
u_{k-1} & \cdots & u_{1} & u_{0} \\
0 & \cdots & 0 & 0 \\
0 & \cdots & 0 & 0 \\
\vdots & \ddots & \vdots & \vdots \\
0 & \cdots & 0 & 0
\end{array}\right), B=\left(\begin{array}{ccccc}
a_{k-1} & 1 & \cdots & 0 & 0 \\
\vdots & \vdots & \ddots & \vdots & \vdots \\
a_{2} & 0 & \cdots & 1 & 0 \\
a_{1} & 0 & \cdots & 0 & 1 \\
a_{0} & 0 & \cdots & 0 & 0
\end{array}\right), C=\left(\begin{array}{ccccc}
0 & 0 & \cdots & 0 & 0 \\
0 & 0 & \cdots & 0 & 0 \\
\vdots & \vdots & \ddots & \vdots & \vdots \\
0 & 0 & \cdots & 0 & 0 \\
0 & 0 & \cdots & 0 & 1
\end{array}\right) .
$$

A straightforward computation shows that the product $A^{m} B^{n} C^{\ell}$ is equal to a matrix where all entries equal zero except for the entry in the upper-right corner which is equal to $u_{k-1}^{m-1} u_{n}$. So, if we assume that $u_{k-1} \neq 0$, then we have the following implications: (1) if $A^{m} B^{n} C^{\ell}=\mathbf{O}_{k, k}$ for some $m, n, \ell \in \mathbb{N}$ with $m, \ell \geq 1$, then $u_{n}=0$; and (2) if $u_{n}=0$, then the equation $A^{m} B^{n} C^{\ell}=\mathbf{O}_{k, k}$ holds for any $m, \ell \geq 1$.

The assumption that $u_{k-1} \neq 0$ is not a serious restriction because we can shift the original sequence by at most $k$ positions to ensure that $u_{k-1} \neq 0$. In other words, instead of $\left(u_{n}\right)_{n=0}^{\infty}$ we can consider a sequence $\left(u_{n+t}\right)_{n=0}^{\infty}$ for some $t>0$. It is easy to check that a 1-LRS of depth $k$ is identically zero if and only if it contains $k$ consecutive zeros. Hence if $\left(u_{n}\right)_{n=0}^{\infty}$ is not identically zero, then we can find $t<k$ such that the sequence $\left(u_{n+t}\right)_{n=0}^{\infty}$ satisfies the condition that $u_{k-1+t} \neq 0$.

Corollary 16. The set of triples $(m, n, \ell)$ that satisfy an equation $A^{m} B^{n} C^{\ell}=\mathbf{O}_{k, k}$ is equal to a finite union of direct products of semilinear sets.

Proof. The corollary follows from Equation (4) above that describes all triples $(m, n, \ell)$ that satisfy the equation $A^{m} B^{n} C^{\ell}=\mathbf{O}_{k, k}$. By construction and the Skolem-Mahler-Lech theorem, the sets $Z_{1}, Z_{2}(m, \ell), Z_{3}(\ell)$ and $Z_{4}(m)$ are semilinear. In Equation (4) we take direct product 
of these sets either with singleton sets or with sets of the form $\mathbb{N}_{k}=\{n \in \mathbb{N}: n \geq k\}$, which are also semilinear sets, and then take a finite union of such products. In other words, Equation (4) can be rewritten as follows

$$
\mathbb{N}_{k} \times Z_{1} \times \mathbb{N}_{k} \bigcup \bigcup_{m, \ell<k}\{m\} \times Z_{2}(m, \ell) \times\{\ell\} \bigcup_{\ell<k} \mathbb{N}_{k} \times Z_{3}(\ell) \times\{\ell\} \bigcup_{m<k}\{m\} \times Z_{4}(m) \times \mathbb{N}_{k} .
$$

The main corollary of Theorem 15 is the following result.

- Corollary 17. The ABC-Z problem is decidable for $3 \times 3$ matrices over algebraic numbers and for $4 \times 4$ matrices over real algebraic numbers.

Proof. By Theorem 15, the ABC-Z problem for $3 \times 3$ matrices over $\mathbf{A}$ is equivalent to the Skolem problem of depth 3 over $\mathbf{A}$, and the ABC-Z problem $4 \times 4$ matrices over $\mathbf{A}_{\mathbb{R}}$ is equivalent to the Skolem problem of depth 4 over $\mathbf{A}_{\mathbb{R}}$. Now the corollary follows from the fact that the Skolem problem is decidable for linear recurrence sequences of depth 3 over $\mathbf{A}$ and of depth 4 over $\mathbf{A}_{\mathbb{R}}[44,33]$.

\section{The $A B C D-Z$ problem in dimension two}

Recall that the ABCD-Z problem in dimension two asks whether there exist natural numbers $k, m, n, \ell \in \mathbb{N}$ such that

$$
A^{k} B^{m} C^{n} D^{\ell}=\mathbf{O}_{2,2} .
$$

In this section we will show that this problem is decidable for $2 \times 2$ upper-triangular matrices with rational coefficients. In the proof we will use the following simple lemmas which show how to diagonalise and compute powers of an upper-triangular $2 \times 2$ matrix.

- Lemma 18. Given an upper triangular matrix $\left(\begin{array}{ll}a & b \\ 0 & c\end{array}\right)$ such that $a \neq c$ then:

$$
\left(\begin{array}{ll}
a & b \\
0 & c
\end{array}\right)=\left(\begin{array}{cc}
1 & -\frac{b}{a-c} \\
0 & 1
\end{array}\right)\left(\begin{array}{ll}
a & 0 \\
0 & c
\end{array}\right)\left(\begin{array}{cc}
1 & \frac{b}{a-c} \\
0 & 1
\end{array}\right)
$$

- Lemma 19. For any matrices of the form $\left(\begin{array}{ll}a & b \\ 0 & c\end{array}\right)$ and $\left(\begin{array}{ll}a & b \\ 0 & a\end{array}\right)$, such that $a \neq c$, and any $k \in \mathbb{N}$ we have $\left(\begin{array}{ll}a & b \\ 0 & c\end{array}\right)^{k}=\left(\begin{array}{cc}a^{k} & b \frac{a^{k}-c^{k}}{a-c} \\ 0 & c^{k}\end{array}\right)$ and $\left(\begin{array}{cc}a & b \\ 0 & a\end{array}\right)^{k}=\left(\begin{array}{cc}a^{k} & k b a^{k-1} \\ 0 & a^{k}\end{array}\right)$.

- Theorem 20. The ABCD-Z problem is decidable for $2 \times 2$ upper-triangular matrices over rational numbers.

Proof. First, note that if one of the matrices $A, B, C$ or $D$ is nilpotent, then Equation (5) obviously has a solution. So from now on we assume that none of $A, B, C$ or $D$ is nilpotent. Furthermore, if $A$ or $D$ is invertible, then the ABCD-Z problem reduces to the ABC-Z problem for rational matrices of dimension two that is decidable by Theorem 15 . So, without loss of generality, we will assume that both $A$ and $D$ are singular matrices.

Now suppose we are given an instance of the ABCD-Z problem which satisfies the abovementioned requirements. We will show that if Equation (5) has a solution, then it has a solution with $k=\ell=1$. Indeed, assume Equation (5) has a solution, and let $(k, m, n, \ell)$ be a solution of minimal length, where the length of a solution is the sum $k+m+n+\ell$. Using 
Theorem 15 we can exclude the case when $k=0$ or $\ell=0$ since in this case our problem is just an instance of the ABC-Z for $2 \times 2$ matrices. So we will assume that in the above solution $k, \ell \geq 1$.

By the Frobenius rank inequality (Theorem 1), we have that:

$$
\operatorname{Rk}\left(A^{k} B^{m} C^{n} D^{\ell-1}\right)+\operatorname{Rk}\left(A^{k-1} B^{m} C^{n} D^{\ell}\right) \leq \operatorname{Rk}\left(A^{k-1} B^{m} C^{n} D^{\ell-1}\right) .
$$

This follows since $\operatorname{Rk}\left(A^{k} B^{m} C^{n} D^{\ell}\right)=\operatorname{Rk}\left(\mathbf{O}_{2,2}\right)=0$. In the above inequality, notice that neither $A^{k} B^{m} C^{n} D^{\ell-1}$ nor $A^{k-1} B^{m} C^{n} D^{\ell}$ is the zero matrix by the assumption that the solution has minimal length. Hence the ranks of the matrices on the left hand side are at least 1. Therefore, $\operatorname{Rk}\left(A^{k-1} B^{m} C^{n} D^{\ell-1}\right)=2$. Since we assumed that $A$ and $D$ are singular, it is necessary that $k=\ell=1$. Also notice that if $\operatorname{Rk}(B)=1$ or $\operatorname{Rk}(C)=1$, then we must have $m=0$ or $n=0$, respectively. Again these cases can be excluded by Theorem 15 .

Thus, using the Frobenius rank inequality and the assumption that the solution is of minimal length, we reduced the ABCD-Z problem to an equation of the form:

$$
A B^{m} C^{n} D=\mathbf{O}_{2,2},
$$

where $\operatorname{Rk}(A)=\operatorname{Rk}(D)=1$ and $\operatorname{Rk}(B)=\operatorname{Rk}(C)=2$.

We assumed that $A$ and $D$ have rank one but are not nilpotent. This means that they have one zero and one nonzero element on the diagonal, in particular, they satisfy the condition of Lemma 18. Hence we can find invertible rational matrices $S_{A}$ and $S_{D}$ such that

$$
A=S_{A}^{-1}\left(\begin{array}{cc}
a & 0 \\
0 & 0
\end{array}\right) S_{A} \quad \text { and } \quad D=S_{D}^{-1}\left(\begin{array}{ll}
d & 0 \\
0 & 0
\end{array}\right) S_{D},
$$

where $a, d$ are nonzero rational numbers. Applying Lemma 14 with matrix $S_{A} B^{m} C^{n} S_{D}^{-1}$ in place of $B$, we conclude that $A B^{m} C^{n} D=\mathbf{O}_{2,2}$ holds if and only if the $(1,1)$-entry of $S_{A} B^{m} C^{n} S_{D}^{-1}$ equals zero. In other words, the equation $A B^{m} C^{n} D=\mathbf{O}_{2,2}$ is equivalent to $s_{m, n}=\mathbf{u}^{\top} B^{m} C^{n} \mathbf{v}=0$, where $\mathbf{u}^{\top}=\mathbf{e}_{1}^{\top} S_{A}$ and $\mathbf{v}=S_{D}^{-1} \mathbf{e}_{1}$ are vectors with rational coefficients.

We will consider three cases: (1) both $B$ and $C$ have distinct eigenvalues, (2) both $B$ and $C$ have a single eigenvalue of multiplicity 2 and (3) one matrix has distinct eigenvalues and the other has a single eigenvalue of multiplicity 2 .

Case (1): $B$ and $C$ have distinct eigenvalues, that is, $B=\left(\begin{array}{cc}b_{1} & b_{3} \\ 0 & b_{2}\end{array}\right)$ and $C=\left(\begin{array}{cc}c_{1} & c_{3} \\ 0 & c_{2}\end{array}\right)$, where $b_{1} \neq b_{2}$ and $c_{1} \neq c_{2}$. By Lemma 19 we have

$$
B^{m}=\left(\begin{array}{cc}
b_{1}^{m} & b_{3} \frac{b_{1}^{m}-b_{2}^{m}}{b_{1}-b_{2}} \\
0 & b_{2}^{m}
\end{array}\right) \quad \text { and } \quad C^{n}=\left(\begin{array}{cc}
c_{1}^{n} & c_{3} \frac{c_{1}^{n}-c_{2}^{n}}{c_{1}-c_{2}} \\
0 & c_{2}^{n}
\end{array}\right)
$$

Multiplying these matrices we obtain: $B^{m} C^{n}=$

$$
=\left(\begin{array}{cc}
b_{1}^{m} & b_{3} \frac{b_{1}^{m}-b_{2}^{m}}{b_{1}-b_{2}} \\
0 & b_{2}^{m}
\end{array}\right)\left(\begin{array}{cc}
c_{1}^{n} & c_{3} \frac{c_{1}^{n}-c_{2}^{n}}{c_{1}-c_{2}} \\
0 & c_{2}^{n}
\end{array}\right)=\left(\begin{array}{cc}
b_{1}^{m} c_{1}^{n} & b_{3} \frac{b_{1}^{m} c_{2}^{n}-b_{2}^{m} c_{2}^{n}}{b_{1}-b_{2}}+c_{3} \frac{b_{1}^{m} c_{1}^{n}-b_{1}^{m} c_{2}^{n}}{c_{1}-c_{2}} \\
0 & b_{2}^{m} c_{2}^{n}
\end{array}\right)
$$

From this formula one can see that the entries of $B^{m} C^{n}$ are linear combinations of $b_{1}^{m} c_{1}^{n}$, $b_{1}^{m} c_{2}^{n}$ and $b_{2}^{m} c_{2}^{n}$ with rational coefficients. Notice that the term $b_{2}^{m} c_{1}^{n}$ does not appear in the entries of $B^{m} C^{n}$. Since $\mathbf{u}$ and $\mathbf{v}$ are rational vectors we conclude that

$$
s_{m, n}=\mathbf{u}^{\top} B^{m} C^{n} \mathbf{v}=\alpha b_{1}^{m} c_{1}^{n}+\beta b_{1}^{m} c_{2}^{n}+\gamma b_{2}^{m} c_{2}^{n},
$$


where $\alpha, \beta, \gamma \in \mathbb{Q}$. Multiplying the equation $s_{m, n}=\alpha b_{1}^{m} c_{1}^{n}+\beta b_{1}^{m} c_{2}^{n}+\gamma b_{2}^{m} c_{2}^{n}=0$ by the product of denominators of $\alpha, \beta, \gamma$ and $b_{1}, b_{2}, c_{1}, c_{2}$ we can rewrite it in the following form

$$
a s^{m} r^{n}+b s^{m} t^{n}+c q^{m} t^{n}=0
$$

where $a, b, c, q, r, s, t$ are integers and $a, b, c$ are relatively prime. Recall that we want to find out if there exist $m, n \in \mathbb{N}$ that satisfy the above exponential Diophantine equation. If one of the coefficients $a, b, c$ is zero, then this problem is easy to solve. For instance, if $b=0$ then the above equation is equivalent to $a s^{m} r^{n}=-c q^{m} t^{n}$. This equality holds if and only if $a s^{m} r^{n}$ and $-c q^{m} t^{n}$ have the same sign and $v_{p}\left(a s^{m} r^{n}\right)=v_{p}\left(-c q^{m} t^{n}\right)$ for every prime divisor $p$ of $a, c, s, r, q$ or $t$. Each of these conditions can be expressed as a linear Diophantine equation. For instance, the sign of $a s^{m} r^{n}$ or $-c q^{m} t^{n}$ depends on the parity of $m$ and $n$. So, the requirement that $a s^{m} r^{n}$ and $-c q^{m} t^{n}$ have the same sign can be written as a linear congruence equation in $m$ and $n$ modulo 2 , which in turn can be expressed as a linear Diophantine equation. Since a system of linear Diophantine equations can be effectively solved, we can find out whether there exist $m$ and $n$ that satisfy $a s^{m} r^{n}=-c q^{m} t^{n}$.

Now suppose that $a, b, c$ are relatively prime nonzero integers. Let $T$ be all primes that appear in $s, r, q$ or $t$. Theorem 5 gives an upper bound on nonzero relatively prime integers $x, y, z$ that are composed of the primes from $T$ and satisfy the equation $a x+b y+c z=0$. Therefore, we can algorithmically compute the following set

$$
\mathcal{U}=\{(x, y, z): a x+b y+c z=0, x, y, z \neq 0 \text { and } \operatorname{gcd}(x, y, z)=1\} .
$$

Next for each triple $(x, y, z) \in \mathcal{U}$ we want to find out if there exist $m, n \in \mathbb{N}$ such that

$$
\left(s^{m} r^{n}, s^{m} t^{n}, q^{m} t^{n}\right)=(x g, y g, z g)
$$

for some $g \in \mathbb{N}$ that is composed of the primes from $T$. It is not hard to see that Equation (6) holds if and only if for every $p \in T$

$$
v_{p}\left(s^{m} r^{n}\right)-v_{p}(x)=v_{p}\left(s^{m} t^{n}\right)-v_{p}(y)=v_{p}\left(q^{m} t^{n}\right)-v_{p}(z)
$$

and $s^{m} r^{n}, s^{m} t^{n}, q^{m} t^{n}$ have the same signs as $x, y, z$, respectively. Since these conditions can be expressed as a system of linear Diophantine equations, we can algorithmically find if there are $m, n \in \mathbb{N}$ that satisfy Equation (6). If such $m$ and $n$ exist for at least one triple $(x, y, z) \in \mathcal{U}$, then the original equation $s_{m, n}=0$ has a solution. Otherwise, the equation $s_{m, n}=0$ does not have a solution.

To finish the proof we also need to consider the following cases:

Case (2): both $B$ and $C$ have a single eigenvalue of multiplicity 2 .

Case (3): one matrix has distinct eigenvalues and the other has a single eigenvalue of multiplicity 2 .

Case (2) is the easiest one in the sense that in this case we can reduce the ABCD-Z problem to a single linear Diophantine equation without using Baker's theorem. In Case (3) we will reduce the ABCD-Z problem to a linear-exponential Diophantine equation of the form $c \frac{s^{m}}{t^{m}}=a+b n$, where $a, b, c, s, t \in \mathbb{Z}, t>0$ and $\operatorname{gcd}(s, t)=1$, which is not hard solve. Again, this case does not require the use of Baker's theorem. All the necessary details for the proof of Cases (2) and (3) are given in the full version [6].

- Remark. It is interesting to note that in Cases (1) and (2) the solutions $(m, n)$ of the equation $s_{m, n}=0$ are described by linear Diophantine equations, and only in Case (3) we have a linear-exponential equation. This agrees with an example from Proposition 23, in which matrix $A$ has a single eigenvalue 1 of multiplicity 2 and matrix $B$ has distinct eigenvalues 1 and 2 . 
- Remark. In the above argument we used Theorem 5 to obtain a bound on the solutions of the equation $a s^{m} r^{n}+b s^{m} t^{n}+c q^{m} t^{n}=0$, which is a special type of an S-unit equation. This leaves open an interesting question of whether any S-unit equation can be encoded into the ABCD-Z problem.

The obvious question is how hard would it be to solve $n$-LRSs, or in general multiplicative matrix equations, in low dimensions. In fact we can show that the Skolem problem for $n$-LRSs of depth 2 is NP-hard. It is not direct but an easy corollary following the hardness proof of the mortality problem for $2 \times 2$ matrices [4].

- Theorem 21 ([4]). The mortality problem for integer matrices of dimension two is NP-hard.

- Corollary 22. Determining if the zero set of an $n$-LRS of depth 2 is empty is NP-hard.

Proof. The main idea of the proof is to reuse the NP-hardness result for the mortality problem in $2 \times 2$ integral matrices from [4] by replacing the simulation of alternatives in subset sum problem defined by a state structure (which is guaranteed by specific order of unique cancellations) by the equation structure in which avoiding alternatives corresponds to the elements in the equation with zero power. See the formal construction in the full version [6].

Another interesting observation is that the zero set of a 2-LRS is not necessarily semilinear, in contrast to the situation for 1-LRSs, which indicates that the Skolem problem for 2-LRSs is likely to be significantly harder than the Skolem problem for 1-LRSs even for sequences of small depth.

- Proposition 23. There exists a 2-LRS of depth 2 for which the zero set is not semilinear.

Proof. Let $u=(0,1)^{T}, v=(1,-1)^{T}, A=\left(\begin{array}{ll}1 & 0 \\ 1 & 1\end{array}\right)$ and $B=\left(\begin{array}{ll}1 & 0 \\ 0 & 2\end{array}\right)$. Define

$$
s_{n, m}=u^{T} A^{n} B^{m} v=(0,1)\left(\begin{array}{ll}
1 & 0 \\
1 & 1
\end{array}\right)^{n}\left(\begin{array}{ll}
1 & 0 \\
0 & 2
\end{array}\right)^{m}\left(\begin{array}{c}
1 \\
-1
\end{array}\right)=n-2^{m} \text {. }
$$

Then $s_{n, m}=0$ if and only if $n=2^{m}$. Clearly, the zero set is not semilinear.

\section{References}

1 L. Babai, R. Beals, J-Y. Cai, G. Ivanyos, and E. M. Luks. Multiplicative equations over commuting matrices. In Proc. of the Seventh Annual ACM-SIAM Symposium on Discrete Algorithms, SODA 96, 1996.

2 C. Baier, S. Kiefer, J. Klein, S. Klüppelholz, D. Müller, and J. Worrell. Markov Chains and Unambiguous Büchi Automata. In Computer Aided Verification - 28th International Conference, CAV 2016, Toronto, ON, Canada, July 17-23, 2016, Proceedings, Part I, pages 23-42, 2016.

3 P. C. Bell, V. Halava, T. Harju, J. Karhumäki, and I. Potapov. Matrix equations and Hilbert's tenth problem. International Journal of Algebra and Computation, 18:1231-1241, 2008.

4 P. C. Bell, M. Hirvensalo, and I. Potapov. Mortality for $2 \times 2$ matrices is NP-hard. In Mathematical Foundations of Computer Science (MFCS 2012), volume LNCS 7464, pages 148-159, 2012.

5 P. C. Bell, M. Hirvensalo, and I. Potapov. The identity problem for matrix semigroups in $\mathrm{SL}_{2}(\mathbb{Z})$ is NP-complete. In Proceedings of the Annual ACM-SIAM Symposium on Discrete Algorithms (SODA'17), pages 187-206, 2017. 
6 Paul C. Bell, Igor Potapov, and Pavel Semukhin. On the Mortality Problem: from multiplicative matrix equations to linear recurrence sequences and beyond. CoRR, abs/1902.10188, 2019. arXiv: 1902.10188.

7 V. Blondel, E. Jeandel, P. Koiran, and N. Portier. Decidable and undecidable problems about quantum automata. SIAM Journal on Computing, 34:6:1464-1473, 2005.

8 V. D. Blondel, O. Bournez, P. Koiran, C. Papadimitriou, and J. N. Tsitsiklis. Deciding stability and mortality of piecewise affine dynamical systems. Theoretical Computer Science, 255(1-2):687-696, 2001.

9 V. D. Blondel and N. Portier. The presence of a zero in an integer linear recurrent sequence is NP-hard to decide. Linear Algebra and its Applications, pages 91-98, 2002.

10 V. D. Blondel and J. N. Tsitsiklis. Complexity of stability and controllability of elementary hybrid systems. Automatica, 35:479-489, 1999.

11 Jin-yi Cai, Wolfgang H. J. Fuchs, Dexter Kozen, and Zicheng Liu. Efficient Average-Case Algorithms for the Modular Group. In 35th Annual Symposium on Foundations of Computer Science, Santa Fe, New Mexico, USA, 20-22 November 1994, pages 143-152, 1994.

12 Jin-yi Cai, Richard J. Lipton, and Yechezkel Zalcstein. The Complexity of the Membership Problem for 2-generated Commutative Semigroups of Rational Matrices. In 35th Annual Symposium on Foundations of Computer Science, Santa Fe, New Mexico, USA, 20-22 November 1994, pages 135-142, 1994.

13 J. Cassaigne, V. Halava, T. Harju, and F. Nicolas. Tighter Undecidability Bounds for Matrix Mortality, Zero-in-the-Corner Problems, and More. CoRR, abs/1404.0644, 2014.

14 V. Chonev, J. Ouaknine, and J. Worrell. On the Complexity of the Orbit Problem. Journal of the ACM, 63(3):1-18, 2016.

15 Ventsislav Chonev, Joël Ouaknine, and James Worrell. On the Skolem Problem for Continuous Linear Dynamical Systems. In 43rd International Colloquium on Automata, Languages, and Programming, ICALP 2016, July 11-15, 2016, Rome, Italy, pages 100:1-100:13, 2016.

16 J.-H. Evertse, K. Győry, C. L. Stewart, and R. Tijdeman. $S$-unit equations and their applications. In New advances in transcendence theory (Durham, 1986), pages 110-174. Cambridge Univ. Press, Cambridge, 1988.

17 E. Galby, J. Ouaknine, and J. Worrell. On matrix powering in low dimensions. In 32nd International Symposium on Theoretical Aspects of Computer Science (STACS'15), pages 329-340, 2015.

18 K. Győry. On the abc conjecture in algebraic number fields. Acta Arith., 133(3):281-295, 2008.

19 V. Halava, T. Harju, M. Hirvensalo, and J. Karhumäki. Skolem's problem - on the border between decidability and undecidability. In TUCS Technical Report Number 683, 2005.

20 G. Hansel. Une démonstration simple du théorème de Skolem-Mahler-Lech. Theoret. Comput. Sci., 43(1):91-98, 1986.

21 Michael A. Harrison. Lectures on Linear Sequential Machines. Academic Press, Inc., Orlando, FL, USA, 1969.

22 K. Hoffman and R. Kunze. Linear algebra. Second edition. Prentice-Hall, Inc., Englewood Cliffs, N.J., 1971.

23 Juha Honkala. Products of matrices and recursively enumerable sets. Journal of Computer and System Sciences, 81(2):468-472, 2015.

24 R. Horn and C. Johnson. Matrix Analysis. Cambridge University Press, 1990.

25 Ravindran Kannan and Richard J. Lipton. The Orbit Problem is Decidable. In Proceedings of the Twelfth Annual ACM Symposium on Theory of Computing, STOC '80, pages 252-261, New York, NY, USA, 1980. ACM.

26 J.-Y. Kao, N. Rampersad, and J. Shallit. On NFAs where all states are final, initial, or both. Theor. Comput. Sci., 410(47-49):5010-5021, 2009.

27 S.-K. Ko, R. Niskanen, and I. Potapov. On the Identity Problem for the Special Linear Group and the Heisenberg Group. In 45th International Colloquium on Automata, Languages, and 
Programming, ICALP 2018, July 9-13, 2018, Prague, Czech Republic, pages 132:1-132:15, 2018.

28 Daniel König, Markus Lohrey, and Georg Zetzsche. Knapsack and subset sum problems in nilpotent, polycyclic, and co-context-free groups. CoRR, abs/1507.05145, 2015. arXiv: 1507.05145.

29 C. Lech. A note on recurring series. Ark. Mat. 2, 1953.

30 Alexei Lisitsa and Igor Potapov. Membership and Reachability Problems for Row-Monomial Transformations. In Jiří Fiala, Václav Koubek, and Jan Kratochvíl, editors, Mathematical Foundations of Computer Science 2004, pages 623-634, Berlin, Heidelberg, 2004. Springer Berlin Heidelberg.

31 Markus Lohrey. Rational subsets of unitriangular groups. IJAC, 25(1-2):113-122, 2015.

32 K. Mahler. Eine arithmetische Eigenschaft der Taylor-Koeffizienten rationaler Funktionen. In Akad. Wet. Amsterdam 38, pages 50-60, 1935.

33 M. Mignotte, T. N. Shorey, and R. Tijdeman. The distance between terms of an algebraic recurrence sequence. J. Reine Angew. Math., 349:63-76, 1984.

34 C. Nuccio and E. Rodaro. Mortality Problem for $2 \times 2$ Integer Matrices. In SOFSEM 2008: Theory and Practice of Computer Science, 34th Conference on Current Trends in Theory and Practice of Computer Science, Nový Smokovec, Slovakia, January 19-25, 2008, Proceedings, pages 400-405, 2008. doi:10.1007/978-3-540-77566-9_34.

35 J. Ouaknine, J. Sousa Pinto, and J. Worrell. On termination of integer linear loops. In Proceedings of the Twenty-Sixth Annual ACM-SIAM Symposium on Discrete Algorithms (SODA 2015), pages 957-969, 2015.

36 J. Ouaknine, A. Pouly, J. Sousa-Pinto, and J. Worrell. Solvability of Matrix-Exponential Equations. In Proceedings of the 31st Annual ACM/IEEE Symposium on Logic in Computer Science (LICS'16), pages 798-806, 2016.

37 Joël Ouaknine and James Worrell. Decision Problems for Linear Recurrence Sequences. In Reachability Problems - 6th International Workshop, RP 2012, Bordeaux, France, September 17-19, 2012. Proceedings, pages 21-28, 2012.

38 Joël Ouaknine and James Worrell. On the Positivity Problem for Simple Linear Recurrence Sequences,. In Automata, Languages, and Programming - 41st International Colloquium, ICALP 2014, Copenhagen, Denmark, July 8-11, 2014, Proceedings, Part II, pages 318-329, 2014.

39 Joël Ouaknine and James Worrell. Positivity Problems for Low-Order Linear Recurrence Sequences. In Proceedings of the Twenty-Fifth Annual ACM-SIAM Symposium on Discrete Algorithms, SODA 2014, Portland, Oregon, USA, January 5-7, 2014, pages 366-379, 2014.

40 M. S. Paterson. Unsolvability in $3 \times 3$ matrices. Studies in Applied Mathematics, 49(1):105-107, 1970 .

41 Igor Potapov and Pavel Semukhin. Decidability of the Membership Problem for $2 \times 2$ integer matrices. In Proceedings of the Twenty-Eighth Annual ACM-SIAM Symposium on Discrete Algorithms, SODA 2017, Barcelona, Spain, Hotel Porta Fira, January 16-19, pages 170-186, 2017.

42 Igor Potapov and Pavel Semukhin. Membership Problem in GL(2, Z) Extended by Singular Matrices. In 42nd International Symposium on Mathematical Foundations of Computer Science, MFCS 2017, August 21-25, 2017 - Aalborg, Denmark, pages 44:1-44:13, 2017.

43 T. Skolem. Ein Verfahren zur Behandlung gewisser exponentialer Gleichungen und diophantischer Gleichungen. Skand. Mat. Kongr., 8:163-188, 1934.

44 N. K. Vereshchagin. The problem of the appearance of a zero in a linear recursive sequence. Mat. Zametki 38, 347(2):609-615, 1985. 